\title{
Helmut Glück, Mark Häberlein, Andreas Flurschütz da Cruz (Hg.), Adel und Mehrsprachigkeit in der Frühen Neuzeit
}

\section{Thomas Nicklas}

\section{(2) OpenEdition \\ Journals}

Édition électronique

URL : http://journals.openedition.org/ifha/10722

DOI : 10.4000/ifha.10722

ISSN : 2198-8943

Éditeur

IFRA - Institut franco-allemand (sciences historiques et sociales)

Référence électronique

Thomas Nicklas, «Helmut Glück, Mark Häberlein, Andreas Flurschütz da Cruz (Hg.), Adel und Mehrsprachigkeit in der Frühen Neuzeit », Revue de l'IFHA [En ligne], Date de recension, mis en ligne le 04 mai 2020, consulté le 24 septembre 2020. URL : http://journals.openedition.org/ifha/10722 ; DOI : https://doi.org/10.4000/ifha.10722

Ce document a été généré automatiquement le 24 septembre 2020

(CIFHA 


\section{Helmut Glück, Mark Häberlein,} Andreas Flurschütz da Cruz (Hg.), Adel und Mehrsprachigkeit in der Frühen Neuzeit

Thomas Nicklas

\section{RÉFÉRENCE}

Helmut Glück, Mark Häberlein, Andreas Flurschütz da Cruz (Hg.), Adel und Mehrsprachigkeit in der Frühen Neuzeit. Ziele, Formen und Praktiken des Erwerbs und Gebrauchs von Fremdsprachen, Wiesbaden: Harrassowitz Verlag 2019, 259 p., $58 €$ 
L'histoire de l'apprentissage des langues étrangères et de l'évolution $\mathrm{du}$ plurilinguisme à travers les époques est un champ de recherche relativement nouveau, situé à l'intersection de l'histoire, de la linguistique et de la didactique des langues. Dans l'espace germanophone, la société Matthias Kramer, créée en 2013 et rattachée à l'université de Bamberg, vise à coordonner et à encourager les recherches dans ce domaine (MatthiasKramer-Gesellschaft zur Erforschung der Geschichte des Fremdsprachenerwerbs und der Mehrsprachigkeit). Cette association porte le nom d'un important linguiste allemand. Après avoir appris l'italien et l'espagnol à la cour polyglotte de

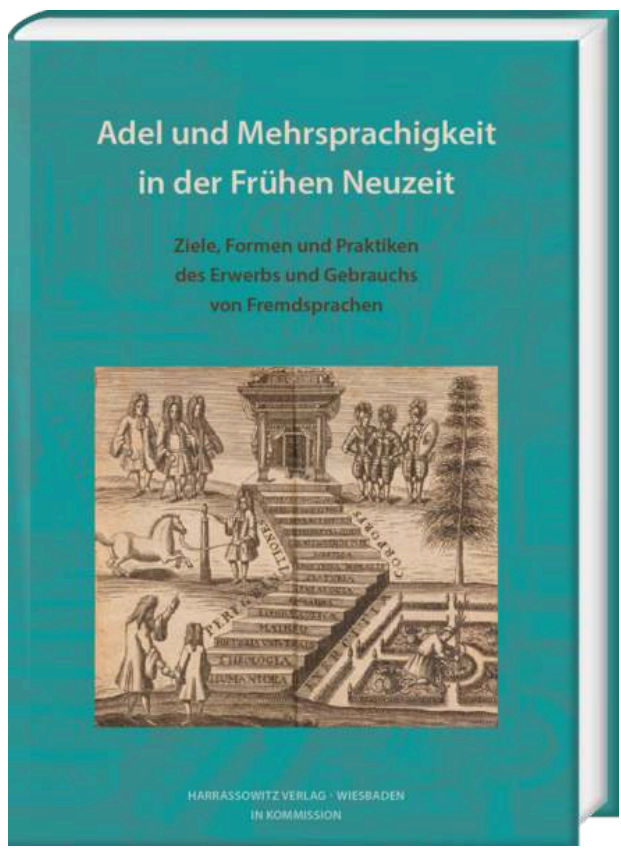
l'empereur Léopold I ${ }^{\mathrm{er}}$, Matthias Kramer (1640-1729) s'installa à Nuremberg où il exerça comme maître de langues, tout en publiant bon nombre de dictionnaires. En le choisissant comme patron, cette association accentue l'intérêt marqué qu'elle porte au plurilinguisme comme facteur culturel important de l'époque moderne.

Issu d'un colloque organisé en 2016 à la Bibliotheca Augusta de Wolfenbüttel, ce volume sur la noblesse et l'apprentissage des langues étrangères couvre toute la période étudiée. Castiglione, dans son Livre du courtisan publié en 1528 (première traduction française de 1537), avait souligné l'importance de cultiver les langues, puisqu'un homme de la cour devait maîtriser l'art de la conversation élégante en plusieurs idiomes. Les idées de Castiglione faisaient écho à une pratique déjà bien ancrée dans le monde des cours italiennes et germaniques. On voit donc les jeunes princes des grandes dynasties du Saint Empire de la première moitié du XVI ${ }^{\mathrm{e}}$ siècle partir pour la France afin d'acquérir une certaine connaissance de la langue, à l'instar du jeune Louis $\mathrm{V}$ du Palatinat qui séjourna à la cour de France entre 1502 et 1504, sans faire toutefois preuve d'un vrai talent pour la maîtrise des langues étrangères, d'après Benjamin Müsegades (Heidelberg). Pour des raisons diplomatiques et dynastiques, toute une génération de princes dans l'empire de Charles Quint, appartenant aux maisons de Wittelsbach, de Wettin (Saxe) et de Wurtemberg, étaient versés dans la langue française, sans qu'on ait beaucoup d'informations sur leurs modes d'acquisition des connaissances linguistiques. Il importe donc d'étudier les cours princières comme lieux de diffusion d'un savoir sur les langues et d'une maîtrise par l'oral et par l'écrit de l'idiome étranger.

Par conséquent, Elena Taddei (Innsbruck) s'attache à l'étude de la cour splendide des princes d'Este à Ferrare, au XVI ${ }^{\mathrm{e}}$ siècle, qui entretenait des liens étroits avec le monde germanique, notamment avec les Habsbourg de Vienne et d'Innsbruck, les Wittelsbach de Munich et les Wettin de Dresde. Alphonse II d'Este, cinquième (et dernier) duc de Ferrare, avait épousé la sœur de l'empereur Maximilien II, Barbara, à qui il écrivait des lettres en italien et en allemand. Fils d'une princesse française (Renée de France), le duc refusa toutefois de s'exprimer en français face à des visiteurs étrangers. En 1581, 
Alphonse reçut à sa cour un illustre voyageur français, Michel de Montaigne, à qui il ne parlait qu'en italien. On voit donc quel est le degré de complexité de la thématique, étant donné que ces nobles ne désiraient pas toujours entretenir leur connaissance de la langue. Le "désapprentissage » linguistique fait aussi partie du périmètre de cette recherche sur la pratique des langues dans les cours de l'époque moderne. Dörthe Buchhester (Hildesheim) analyse «la question des langues " pour une autre cour du $\mathrm{XVI}^{\mathrm{e}}$ siècle, située bien loin de l'Italie, celle des ducs de Poméranie, à Stettin, au bord de la mer Baltique. Les jeunes princes poméraniens parlaient bas-allemand, mais la langue de l'écrit à la cour de Stettin était de plus en plus souvent le haut-allemand. En plus, on demandait aux jeunes membres de la famille ducale d'apprendre le polonais, en raison de la proximité géographique et des liens dynastiques. Mais on ne s'arrêtait pas là, l'apprentissage du latin érudit et du français courtisan étant également souhaitable.

Une autre approche du phénomène investit les parcours individuels. L'archiviste Nils Jörn (Wismar) étudie le cas particulier d'un président de tribunal suédois de Wismar, dans la partie du Mecklembourg rattachée à la couronne de Suède après les traités de Westphalie, en 1648. Il s'agit d'un certain Gustav Helmfel(d)t, fils d'un feld-maréchal suédois, choisi notamment à cause de son excellente maîtrise des langues étrangères pour ce poste important. Mais le jeune talent qui venait d'être nommé comme magistrat à Wismar partit tout de suite en mission diplomatique pour la Pologne (pays dont il parlait parfaitement la langue) où ce personnage surdoué mourut subitement, en laissant vacante la place taillée à sa mesure, celle d'un magistrat maniant les langues du droit et de la diplomatie totius maris baltici. John Gallagher (Leeds) suit les traces d'un autre jeune homme, d'origine anglaise, également promis à une belle carrière, Philippe Perceval, parcourant le continent entre 1676 et 1679, et dont le périple est très bien documenté dans les archives. Un seul article compris dans le volume adopte une tout autre perspective, en ne s'intéressant pas aux apprenants, mais aux enseignants de langues. Andrea Bruschi (Vérone) présente les interprètes du roi, groupe de traducteurs et de maîtres de langue, dont les capacités pédagogiques furent appréciées par la noblesse française, en quête d'enseignants pour la formation de leurs enfants.

Martin Holy (Prague) et Andreas Florschütz da Cruz (Bamberg) traitent quant à eux de l'éducation, notamment linguistique, des jeunes nobles des pays tchèques et de l'Allemagne du Sud. Au XVII ${ }^{\mathrm{e}}$ siècle, le Grand Tour (Kavalierstour) avait, comme on le sait, pour but de parfaire les arts de la cour, dont la maîtrise des langues étrangères, des jeunes gens de la noblesse. Contrairement aux Bildungsreisen des classes bourgeoises au $\mathrm{XIX}^{\mathrm{e}}$ siècle, ces pérégrinations ne visaient pas à se forger une culture individuelle, mais à participer à une culture aristocratique commune européenne, dont un canon de capacités linguistiques faisait partie. L'importance du latin, normalement peu prisé par la noblesse, s'estompait en faveur des langues vernaculaires modernes, français et italien en tête. Il en alla tout autrement en Croatie, partie intégrante du royaume de Hongrie, où les élites restaient très attachées au latin, «langue nationale » de la vie administrative et politique. Ivana Horbec et Maja Matasovic (Zagreb) montrent très bien comment le latin résistait et se maintenait dans les territoires croates face au hongrois de la noblesse magyare et contre l'allemand des fonctionnaires habsbourgeois, au XVIII siècle, tout en créant des symbioses culturelles avec les espaces germano- ou italophones. Ineta Balode (Riga) s'intéresse aux élites des territoires baltes, rattachés à la Russie de Pierre le Grand par le biais du traité de Nystad, en 1721, en répondant à la question de savoir comment la noblesse de culture germanique de ces régions se situa par rapport aux trois langues qui dominaient 
désormais le champ politique et culturel (allemand, français, russe). Vladislav Rjéoutski (Moscou) expose le début de l'apprentissage du français en Russie à l'époque de Pierre le Grand, en s'intéressant aux deux capitales de l'Empire russe, Moscou et SaintPétersbourg, où la nouvelle grande puissance européenne forma ses futurs diplomates.

Finalement, deux exposés se penchent sur le plurilinguisme au féminin, phénomène assez répandu au sein de la noblesse des XVII et $\mathrm{XVIII}^{\mathrm{e}}$ siècles. Barbara Kaltz (Aix-enProvence) étudie les pratiques de l'apprentissage du français et de l'italien de la part des jeunes femmes nobles en Allemagne et en Angleterre. Helga Meise (Reims) présente la duchesse Caroline de Deux-Ponts (1704-1774) et ses deux filles, grandes bibliophiles possédant de belles bibliothèques richement dotées en ouvrages d'expression française. Dans leurs lectures, dans leurs conversations et dans leur écriture épistolaire, ces princesses allemandes accordèrent une toute première place au français, confirmant ainsi le rang de l'idiome international de l'Europe cultivée, polie et « éclairée ».

Tous les textes de l'ouvrage sont en allemand, à l'exception de ceux de John Gallagher et d'Andrea Bruschi qui sont en anglais. Des résumés anglais et français des articles auraient sans doute contribué à la diffusion de ces recherches et bien servi la cause de la société Matthias Kramer, résolument tournée vers l'histoire du plurilinguisme. La panoplie de données et les éléments d'analyse que réunit ce recueil riche et intéressant appelleraient une conclusion qui relie les fils, tout en ouvrant des perspectives pour d'autres recherches dans ce champ novateur qui reste encore largement à défricher.

\section{INDEX}

Index chronologique : Époque moderne (1500-1800)

Thèmes : Histoire de l'apprentissage des langues étrangères, Histoire sociale 